\title{
Individual and community level predictors of maternal timely postnatal care services utilization in Ethiopia: A multilevel-mixed effects analysis
}

Afework Tadele ( $\square$ afatadele@gmail.com )

Jimma University https://orcid.org/0000-0001-5682-6117

Masrie Getinet

Jimma University

Research article

Keywords: Postnatal care, multilevel, DHS, Ethiopia

Posted Date: November 25th, 2019

DOl: https://doi.org/10.21203/rs.2.17670/v1

License: (c) (1) This work is licensed under a Creative Commons Attribution 4.0 International License.

Read Full License 
$3 \quad$ Individual and community level predictors of maternal timely postnatal 4 care services utilization in Ethiopia: A multilevel-mixed effects analysis

5

6

Afework Tadele $^{1^{*}}$ Masrie Getinet ${ }^{2 \mathbb{I}}$

${ }^{1}$ Population and Family Health, Jimma University, Jimma, Oromia, Ethiopia

${ }^{2}$ Epidemiology and Biostatistics, Jimma University, Jimma, Oromia, Ethiopia

9

10

11

*1 Corresponding author: e-mail: afatadele@gmail.com (AT)

2II: Co-author: e-mail: masriegetnet16.biostat@gmail.com (MA)

19

20

21

22 
Introduction: Early identification and management of postpartum complications through timely visit for postnatal care is one of the key interventions in reducing maternal mortality. A community-based national level representative dataset was necessary to inform decision makers in achieving the sustainable development goal (SDG) target of reducing maternal mortality by $70 \%$. This study aims to determine the individual and community level predictors of timely utilization of maternal postnatal care service in Ethiopia.

Methods: A community based nationally representative survey of Ethiopian Demographic and Health Survey (EDHS) in 2016 was utilized. A total of 3,948 women aged 15-49 giving birth in the two years before the survey were included. A two-level mixed-effects logistic regression analysis was employed.

Result: Among women aged 15-49 years giving birth in the 2 years before the survey, 17\% had a postnatal check during the first 2 days after birth in Ethiopia. Institutional delivery AOR 2.14 [95\% C.I 1.70, 2.0] and giving birth by caesarean section AOR 1.66 [95\% CI 1.10, 2.50] were found to be an individual level determinants, whereas administrative regions(Oromia 69\%, Somali 56\%, Benishangul 55\%, SNNPR 43\%, Gambela 66\%, Afar 50\% and Dire Dawa $55 \%$ which less likely to utilize postnatal care utilization as compared to Addis Ababa city administrative area), Higher community level wealth AOR 1.44 [95\% C.I 1.08, 1.2], antenatal care coverage AOR 1.52 [95\% C.I 1.19, 1.96] and perceived distance of the health facility as a big problem AOR 0.78 [95\% C.I 0.60, 0.99] were the community level predictors of timely utilization of post-natal care in Ethiopia.

Conclusion: Less than one in five women utilized postnatal care in the first 2 days after birth in Ethiopia. Individual level factors (being gave birth at health facility and caesarean delivery) and community level factors (administrative regions, community level wealth, antenatal care and perception of distance to health facility as a big problem) were found to be significant predictors.

Keywords: Postnatal care; multilevel; DHS; Ethiopia 
Every day in 2017, approximately 810 women die from preventable causes related to pregnancy and childbirth. Moreover, 94\% of all maternal deaths occur in low and lower middle-income countries[1]. Postnatal period is a critical period in the lives of mothers. Yet, this is the most neglected period for the provision of quality care[2]. More than $60 \%$ of maternal deaths occurred in the postpartum period, of these more than $80 \%$ of the maternal death occurs within two weeks of delivery in both developing countries and the United States[3].

According to the World Health Organization(WHO), the postnatal period begins immediately after childbirth and lasts six weeks[4]. On the one hand, postnatal care services are an essential element of the continuum of care for maternal and child health, and it is a bridge in the continuum of care for child health services and family planning services. Woman and her partner/family require more information than they usually receive on care of the baby and mother within the first week after childbirth. On other hand, mothers who didn't received any perinatal care was associated with neonatal complications at six weeks after birth[5].

Although there is a good improvement in maternal and child health care services coverage recently, still postnatal care was only $17 \%$ of received a postnatal check within the first two days of birth in Ethiopia[6]. Still, the experiences and expectations of women and their families should be considered when deciding the timing of postnatal visits. Barriers to the uptake of services and/or access to services also should be considered when deciding on schedules for postnatal care[4].

Studies so far focus on individual level factors associated with post-natal care utilization like residence, antenatal care follow-up, maternal occupation, awareness about problems of postnatal period, institutional delivery, [7-13] marital status[14] decision-making styles, household distances from health institutions, being model family [15] level of education, 
wealth, alive birth outcome of last pregnancy[16, 17], experience of postpartum complications[18] maternal knowledge about postnatal danger signs[19], Mothers' having husband attended formal education, knowledge of postnatal care[20]. However, equity of the postnatal care services will not be addressed with individual level factors, especially in developing country like Ethiopia with diverse sociocultural, and socioeconomic populations. Regression models with individuals as the units of analysis that include both group/community level and individual level variables as predictors of individual level outcomes will provide contextual effects of explanatory variables on response variables. The multilevel effect data analysis utilized in this study was essential to identify both individual and community level barriers of postnatal care. Thus, it is helpful for effective national level design and improvement of existing policies, and strategies for contextualized interventions.

The findings of the study were also decisive in achieving SDG, the global target of reducing maternal mortality ratio to less than 70 per 100,000 births. In addition, it was a vital for the global strategy, aimed at ending preventable maternal mortality through enlightening predictors of inequalities in access to and quality of maternal, and newborn health care services in different clusters of the Ethiopian community[1].

\section{Methods}

\section{Study setting}

Ethiopia is the second populous country in Africa next to Nigeria bordered by Eretria, South Sudan, Sudan, Djibouti and Somalia with a total of more than one hundred ten million people[21]. Ethiopia has a decentralized three-tier system of primary, secondary and tertiary care. The primary level health cares are district hospitals, health centers and their satellite 
health posts; the secondary level health cares are general hospitals and the tertiary level health care are specialized hospitals[22]. Administratively, Ethiopia is divided into nine geographical regions (Tigray, Afar, Amhara, Oromia, Somali, Benishangul-Gumuz, SNNPR, Gambella and Harari) and two administrative cities, Addis Ababa and Diredawa. The 2016 EDHS was a population-based cross-sectional study conducted from January 18, 2016 to June 27, 2016, across the country[6]

\section{Data Source}

In this study the dataset was obtained from MEASURE DHS database at http://dhsprogram.com/data/. The DHS program office gave an authorization letter to access the 2016 EDHS, which is the fourth comprehensive survey. The 2016 EDHS sample was selected in two stages. In the first stage, total of 645 clusters (202 in urban and 443 in rural) were selected randomly proportional to the household size from the sampling strata and in the second stage, 28 households per cluster were selected using systematic random sampling[6]. Representative samples of 18008 households were selected and 16, 650 households were interviewed in 2016 EDHS. For individual interview, 16, 583 eligible women were identified from the interviewed household. Interviews were completed with 15,683 women aged 15-49 years [22]. In this study, among women aged 15-49 years, 3984 gave birth in the two years before the survey were included.

\section{Study variables}

Dependent variable: The outcome variable was binary categorized as "use of postnatal care coded as 1" and "do not use of postnatal care coded as 0 " within 6 weeks or 42 days of delivery. Independent variables: The independent variables for postnatal care use were broadly classified into individual level and community level variables in line with multilevel analytic approach. 
Individual level variables: Educational level of women, household wealth index, perceived distance to health facility to get medical help, employment status of women's, number of ANC visit during pregnancy, delivery by caesarean section, birth order and place of delivery were included as individual level variables.

Community level variables: Aggregated variables at the community level based on the individual information and then the aggregated values were classified as low and high if the median values or the proportions of the clusters were below and above the national value respectively. Based on this, community level wealth index, community level ANC coverage, community level women's education, perceived distance to health facility to get medical help at the community level were considered as community level variables.

Region: The EDHS sample was collected from nine regions and two administrative cities. We used these administrative boundaries as community level factor.

Place of residence: It was classified as rural and urban, considered as a community level factor.

Community level education of women: The median value of educational attainment at the national level was 5 years. Thus, the median value of the aggregated clusters below 5 were proportion of perception of distance to health facility to get medical help as a big problem was proportion as a cut-off point after aggregated of it. 
Community level ANC visit: The median value of ANC visit at the national level was 3 . Hence, the median value of the cluster less than 3 ANC visit was classified as low and greater than or equal to 3 ANC visits were classified as high after aggregated the ANC visit.

\section{Data analysis}

Two level mixed effects logistic regression analyses were employed using STATA version 14. Since, 2016 EDHS data was hierarchical, i.e., individuals (women) were nested in household and household were nested in cluster. The unit of analysis for the characteristics of communitylevel factors was the cluster. For this study, we included 645 clusters in which all the women whose most recent birth was within two years preceding the survey resides.

First, bi-variable two-level mixed effect logistic regression analyses were done to assess the association between the independent variables and dependent variable of the study. The overall categorical variables with a p-value of $<0.25$ at the bivariate two-level mixed effect logistic regression analysis were included into the final model of multivariable two-level mixed effect logistic regression model in which odds ratio with $95 \%$ confidence intervals were estimated to identify the independent variables of institutional delivery. P-values less than 0.05 were employed to declare the statistical significance. Fixed effect and random effect were calculated to assess the individual and cluster variations respectively. Moreover, the frequency table were displayed for the individual and community level variables. All analysis was done on weighted data.

In this analysis four models are displayed, null model (model containing no factors), model I (containing only individual factors), model II (containing only community factors) and model III (both individual and community level factors). The fitted model was:

$$
\log \left[\frac{\pi_{i j}}{1-\pi_{i j}}\right]=\beta_{0}+\beta_{1} \mathrm{X}_{1 i j}+\cdots \beta_{n} \mathrm{X}_{n i j}+u o_{j}+e_{i j}
$$


$177 \checkmark \pi_{i j}$ is the probability of women who delivered at health facility

$178 \checkmark \checkmark 1-\pi_{i j}$ the probability of not delivered at health facility

$179 \checkmark \checkmark \quad \beta_{0}$ is log odds of the intercept

$180 \checkmark \beta_{1} \ldots \beta_{n}$ are the amount of effect by the individual and community-level variables

$181 \checkmark \checkmark \quad X_{1} \ldots X_{n}$ are the independent variables at individual and community level

$182 \checkmark u o_{j}$ is the random error at community(cluster) and $\checkmark \quad e_{i j}$ is the random error at the individual level.

184 The intra-class correlation (ICC) was calculated as the proportion of the between cluster variation in the total variation:

$$
I C C=\frac{\operatorname{Var}\left(u_{o j}\right)}{\operatorname{Var}\left(u o_{j}\right)+\pi^{2} / 3}
$$

Where, component, which is $\pi^{2} / 3 \approx 3.29$. calculated by Proportional Change in Variance (PCV) as:

$$
P C V=\frac{V_{e}-V_{m i}}{V e},
$$

Where,

$\checkmark \quad V_{e}$ is the variance in institutional delivery in the null model. 


\section{Results}

Individual level women characteristics of the survey

199

200

201

202

203

204

205

206

207

Majority (61.09\%) of the women of reproductive age in Ethiopia who gave birth in the two years preceding the survey did not attend formal education. More than seventy five percent of the women were unemployed. Still there is great problem of access to health facility in Ethiopia as majority $(61.43 \%)$ of the women perceived distance to health facility to get medical help was big problem. Regarding maternal health services in the last two years only $32.62 \%$ percent of the women attended four and more ante-natal care and $35.6 \%$ percent gave birth in health facility(table 1).

Table 1: Individual variables of women aged 15-49 giving birth in the 2 years before the 2016 EDHS ( $n=3948)$

\begin{tabular}{|c|c|c|c|c|}
\hline $\begin{array}{l}\text { Individual level } \\
\text { variables }\end{array}$ & Categories & Frequency & $\%$ unweighted & $\%$ weighted \\
\hline \multicolumn{5}{|c|}{ Educational level of women } \\
\hline & No education & 2373 & 60.1 & 61.09 \\
\hline & Primary & 1086 & 27.5 & 30.34 \\
\hline & Secondary & 323 & 8.2 & 5.83 \\
\hline & Higher & 166 & 4.2 & 2.74 \\
\hline \multicolumn{5}{|l|}{ Wealth index } \\
\hline & Poorest & 1417 & 35.9 & 23.92 \\
\hline & Poorer & 662 & 16.8 & 22.06 \\
\hline & Middle & 558 & 14.1 & 20.93 \\
\hline & Richer & 496 & 12.6 & 18.53 \\
\hline & Richest & 815 & 20.6 & 14.55 \\
\hline \multicolumn{5}{|c|}{ Perceived distance to health facility to get medical help } \\
\hline & Not big problem & 1779 & 45.1 & 38.57 \\
\hline & Big problem & 2169 & 54.9 & 61.43 \\
\hline \multicolumn{5}{|c|}{ women's employment status } \\
\hline & No & 2958 & 74.9 & 75.23 \\
\hline & Yes & 990 & 25.1 & 24.77 \\
\hline \multicolumn{5}{|c|}{ Number of ANC visit } \\
\hline & $<4$ & 2527 & 64.0 & 67.38 \\
\hline & $4^{+}$ & 1421 & 36.0 & 32.62 \\
\hline \multicolumn{5}{|c|}{ Delivery by caesarean section } \\
\hline & No & 3818 & 96.7 & 97.4 \\
\hline & Yes & 130 & 3.3 & 2.60 \\
\hline \multirow[t]{2}{*}{ Place of delivery } & Home & 2382 & 60.3 & 64.78 \\
\hline & Health facility & 1566 & 39.7 & 35.22 \\
\hline
\end{tabular}


210 More than eighty eight percent of the clusters were from rural areas of Ethiopia. While, sixty 211 percent of them were classified under higher community level wealth status. There is also about $21275 \%$ community level of antenatal care coverage and women's unemployment status revealed. 213 One in three clusters perceived the distance to Health facility to get medical help at the 214 community level is a big problem (table 2).

Table 2: Community level variables of women aged 15-49 giving birth in the 2 years before survey $216 \quad(n=3948)$

\begin{tabular}{|c|c|c|c|c|}
\hline Community level variables & Categories & $\mathrm{n}$ & Unweighted \% & Weighted \% \\
\hline \multirow{11}{*}{$\begin{array}{l}\text { Administrative } \\
\text { regions }\end{array}$} & Tigray & 409 & 10.4 & 6.83 \\
\hline & Afar & 379 & 9.6 & 1.00 \\
\hline & Amhara & 365 & 9.2 & 18.27 \\
\hline & Oromia & 612 & 15.5 & 45.10 \\
\hline & Somali & 521 & 13.2 & 4.21 \\
\hline & Benishangul & 313 & 7.9 & 1.03 \\
\hline & SNNPR & 477 & 12.1 & 20.40 \\
\hline & Gambela & 264 & 6.7 & 0.24 \\
\hline & Harari & 221 & 5.6 & 0.23 \\
\hline & Dire Dawa & 182 & 5.2 & 2.30 \\
\hline & Addis Ababa & 205 & 4.6 & 0.41 \\
\hline \multirow[t]{2}{*}{ Place of residence } & Urban & 767 & 19.4 & 11.34 \\
\hline & Rural & 3181 & 80.6 & 88.66 \\
\hline \multirow[t]{2}{*}{ Community level wealth } & low & 2017 & 51.1 & 40.33 \\
\hline & High & 1931 & 48.9 & 59.67 \\
\hline \multicolumn{5}{|c|}{ Community level ANC coverage } \\
\hline & low & 2687 & 68.1 & 74.95 \\
\hline & High & 1261 & 31.9 & 25.05 \\
\hline \multicolumn{5}{|c|}{ Community level women's employment } \\
\hline & low & 2854 & 72.3 & 75.49 \\
\hline & High & 1094 & 27.7 & 24.51 \\
\hline \multicolumn{5}{|c|}{ Community level women's education } \\
\hline & low & 1874 & 47.5 & 61.43 \\
\hline & high & 1541 & 39.0 & 38.57 \\
\hline \multicolumn{5}{|c|}{$\begin{array}{l}\text { Perceived distance to Health facility } \\
\text { to get medical help at the community level }\end{array}$} \\
\hline & Not big problem & 1506 & 38.1 & 30.02 \\
\hline & Big problem & 2442 & 61.9 & 69.98 \\
\hline
\end{tabular}


A two-level mixed effects logistic regression model was used to analyse the effects of community characteristics and women's individual-level factors in post-natal care services utilization in Ethiopia. As depicted in the empty model, $36.5 \%$ of the total variance in the odds of postnatal care utilization was accounted for by between-cluster/communities variation of characteristics (ICC $=0.365)$. The between-cluster variability declined over successive models, from $36.5 \%$ in the empty model to $7.1 \%$ in individual-level only model, $6.8 \%$ in community-level only model, and $1 \%$ in the combined model. Accordingly, the combined model of individual-level, and community-level factors was selected for determining postnatal care services utilization.

\section{Maternal level predictors of timely postnatal care service utilization}

The details of effect sizes of both individual and community-level factors on odds of postnatal care service utilization are described in Tables 3 and 4. Delivery by caesarean section were independently and significantly associated with postnatal care utilization.

After adjusting for individual and community-level factors, the odds of using postnatal care was 1.66 times OR 1.66 (95\% CI 1.10, 2.50) higher among women gave birth by caesarean section compared to their counter parts. Similarly, women who gave birth at health facility were twice higher odds of using postnatal care OR 2.14 (95\% CI 1.70, 2.70) as compared those delivered at home (table 3). 
Table 3: Multilevel mixed-effect logistic regression results of individual and community level factors associated with timely use of postnatal care in Ethiopia, 2016 EDHS.

\begin{tabular}{|c|c|c|c|c|}
\hline \multirow{3}{*}{$\begin{array}{l}\text { Variables/ } \\
\text { characteristics }\end{array}$} & \multirow{3}{*}{$\begin{array}{l}\text { Null } \\
\text { model }\end{array}$} & Model I & \multirow{2}{*}{$\begin{array}{c}\text { Model II } \\
\begin{array}{c}\text { Community } \\
\text { level } \\
\text { characteristics }\end{array}\end{array}$} & \multirow{2}{*}{$\begin{array}{l}\text { Model III } \\
\text { Individual and } \\
\text { community level } \\
\text { characteristics }\end{array}$} \\
\hline & & $\begin{array}{l}\text { Individual } \\
\text { characteristics }\end{array}$ & & \\
\hline & & OR $(95 \% \mathrm{C} \mathrm{I})$ & OR (95\% C I) & OR $(95 \%$ C I $)$ \\
\hline \multicolumn{5}{|c|}{ Educational level of women } \\
\hline \multicolumn{2}{|c|}{ No education ${ }^{(\text {Ref })}$} & 1 & & \\
\hline \multicolumn{2}{|c|}{ Primary } & $0.97(0.78,1.21)$ & & $0.92(0.73,1.14)$ \\
\hline \multicolumn{2}{|l|}{ Secondary } & $1.15(0.84,1.59)$ & & $1.04(0.75,1.46)$ \\
\hline \multicolumn{2}{|l|}{ Higher } & $1.55(1.02,2.34) *$ & & $1.40(0.92,2.14)$ \\
\hline \multicolumn{5}{|c|}{ Household wealth } \\
\hline \multicolumn{2}{|c|}{ Poorest $^{(\mathrm{ref})}$} & 1 & & \\
\hline \multicolumn{2}{|l|}{ Poorer } & $1.38(1.03,1.85) *$ & & $1.14(0.83,1.56)$ \\
\hline \multicolumn{2}{|l|}{ Middle } & $1.40(1.03,1.90) *$ & & $0.97(0.68,1.38)$ \\
\hline \multicolumn{2}{|l|}{ Richer } & $1.43(1.05,1.97) *$ & & $0.99(0.68,1.43)$ \\
\hline \multicolumn{2}{|c|}{ Richest } & $2.59(1.90,3.53) * * *$ & & $1.29(0.82,2.01)$ \\
\hline \multicolumn{5}{|c|}{$\begin{array}{l}\text { Perceived distance to Health } \\
\text { facility to get medical help }\end{array}$} \\
\hline \multicolumn{2}{|c|}{ Not big problem ${ }^{(\mathrm{ref})}$} & 1 & & \\
\hline \multicolumn{2}{|c|}{ Big problem } & $0.75(0.62,0.91) * *$ & & $1.0(0.79,1.26)$ \\
\hline \multicolumn{5}{|c|}{$\begin{array}{l}\text { Number of ANC visit during } \\
\text { pregnancy }\end{array}$} \\
\hline \multicolumn{2}{|c|}{$<4$ visit $^{\text {(ref) }}$} & 1 & & \\
\hline \multicolumn{2}{|c|}{$4^{+}$Visit } & $1.26(1.04,1.53) *$ & & $0.95(0.76,1.18)$ \\
\hline \multicolumn{5}{|c|}{ Delivery by caesarean section } \\
\hline \multicolumn{2}{|c|}{$\mathrm{No}^{(\mathrm{ref})}$} & 1 & & \\
\hline \multicolumn{2}{|l|}{ Yes } & $1.76(1.18,2.63) * *$ & & $1.66(1.10,2.50) *$ \\
\hline \multicolumn{5}{|c|}{ Place of delivery } \\
\hline \multicolumn{2}{|c|}{ Not at health facility } & 1 & & \\
\hline \multicolumn{2}{|c|}{ Health facility } & $2.84(2.27,3.55) * * *$ & & $2.14(1.70,2.70) * * *$ \\
\hline
\end{tabular}

\section{Community level predictors of timely postnatal care service utilization}

There is significant administrative regional variation in postnatal care use. The conspicuous observation to emerge from the data comparison was in Oromia 69\%, Somali 56\%, Benishangul 55\%, SNNPR 43\%, Gambela 66\%, Afar 50\% and Dire Dawa 55\% which less likely to utilize postnatal care utilization as compared to Addis Ababa city administrative area. While there is no significant difference between residents of Harari city, Amhara regional state and Tigray regional state as compared to Addis Ababa city. Community level wealth and 
antenatal care coverage were also found to be significant determinants of postnatal care

251

252

253

254

255

256

257

utilization. Community level wealth was 1.4 times OR 1.44 (95\% C.I 1.08, 1.2) and community level antenatal care coverage were 1.5 times OR 1.52 (95\% C.I 1.19, 1.96) more likely to use post-natal care utilization. Perceived community level distance of health facility as a big problem was found to be highly significant determinants of postnatal care. A community who perceived the distance to health facility as a big problem were $22 \%$ less likely OR 0.78 (95\% C.I $0.60,0.99$ ) to utilize postnatal care as compared to their counter parts (table 4).

Table 4: Multilevel mixed-effect logistic regression results of individual and community level predictors of postnatal care in Ethiopia, 2016.

\begin{tabular}{lllcc} 
& Null & Model I & Model II & Model III \\
\cline { 4 - 6 } Variables/ characteristics & $\operatorname{model}$ & $\begin{array}{l}\text { Individual } \\
\text { characteristics }\end{array}$ & $\begin{array}{c}\text { Community level } \\
\text { characteristics }\end{array}$ & $\begin{array}{c}\text { Individual and } \\
\text { community level } \\
\text { characteristics }\end{array}$
\end{tabular}

\section{Administrative regions}

\begin{tabular}{|c|c|c|}
\hline Tigray & $1.31(0.82,2.10)$ & $1.35(0.88,2.07)$ \\
\hline Afar & $0.38(0.21,0.68) * *$ & $0.50(0.29,0.87) *$ \\
\hline Amhara & $0.49(0.29,0.83) * *$ & $0.63(0.39,1.03)$ \\
\hline Oromia & $0.24(0.14,0.42) * * *$ & $0.31(0.19,0.50) * * *$ \\
\hline Somali & $0.32(0.18,0.56) * * *$ & $0.44(0.26,0.73) * *$ \\
\hline Benishangul & $0.36(0.210 .63) * * *$ & $0.45(0.28,0.79) * *$ \\
\hline SNNPR & $0.47(0.29,0.78) * *$ & $0.57(0.36,0.90) * *$ \\
\hline Gambela & $0.27(0.15,0.48) * * *$ & $0.34(0.20,0.58) *$ \\
\hline Harari & $1.06(0.62,1.80)$ & $1.10(0.68,1.79)$ \\
\hline Dire Dawa & $0.49(0.29,0.83) * *$ & $0.55(0.34,0.89) *$ \\
\hline Addis Ababa ${ }^{(\mathrm{ref})}$ & 1 & \\
\hline \multicolumn{3}{|l|}{ Place of residence } \\
\hline $\mathrm{Urban}^{(\mathrm{ref})}$ & 1 & \\
\hline Rural & $0.60(0.44,0.82) * *$ & $0.94(0.65,1.37)$ \\
\hline \multicolumn{3}{|l|}{ Community level wealth } \\
\hline $\operatorname{low}^{(\mathrm{ref})}$ & 1 & \\
\hline High & $1.60(1.21,2.13) * *$ & $1.44(1.08,1.2) *$ \\
\hline \multicolumn{3}{|c|}{ Community level ANC coverage } \\
\hline low ${ }^{\text {(ref) }}$ & 1 & \\
\hline High & $1.73(1.34,2.25) * * *$ & $1.52(1.19,1.96) * *$ \\
\hline \multicolumn{3}{|c|}{ Community level perceptions of distance to HF } \\
\hline Not big problem ${ }^{(\mathrm{ref})}$ & 1 & \\
\hline Big problem & $0.70((0.54,0.90) * *$ & $0.78(0.60,0.99) * *$ \\
\hline
\end{tabular}




\begin{tabular}{rllll}
\hline Random effects & Model 0 & Model 1 & Model 2 & Model 3 \\
\hline \multicolumn{1}{c}{ ICC (\%) } & $36.5 \%$ & $7.1 \%$ & $6.8 \%$ & $1 \%$ \\
\hline \multicolumn{1}{c}{ PCV } & Reference & $81 \%$ & $81.4 \%$ & $97 \%$ \\
\hline $\begin{array}{l}\text { Model fitness } \\
\text { Log likelihood }\end{array}$ & -1883.6 & -1736.0 & -1633.2 & -1536.0 \\
\hline
\end{tabular}

261

262 Discussion

263 Postnatal care is an indispensable intervention in safe motherhood through early identification

264 and management complications which is fatal for the mother and new-borns. Although Ethiopia

265 recently has a great achievement in maternal and child mortality reduction, still there is high

266 maternal mortality ratio and perinatal mortality rate largely due to low postnatal care coverage

267 in which large proportion of maternal and child mortality occurs. Therefore, identifying

268 individual characters and community level determinants of postnatal care has a great

269 contribution designing different interventions for improving maternal and child health.

270 This study found different individual and community-level determinants of postnatal care service utilization were identified. In this study $36.5 \%$ of the total variance in the odds of postnatal care utilization was accounted for the characteristics between-cluster variation. While

$7.1 \%$ in individual-level and $6.8 \%$ in community-level variation were identified. We found much higher values than a cross-sectional study in remote and poorest rural communities of Zambia in 2012, which revealed 22\% and study in Nigeria $10.35 \%$ [23], variance in the use of PNC within 48 hours were attributable to the variations across community clusters[24]. This implies the issues of equity in postnatal care services for availability and accessibility were still great challenge for community.

This study found that women who gave birth by caesarean section were 1.66 times higher odds of using postnatal care services within the first two days compared to those gave birth by another mode of delivery. This is in a good agreement with the study in rural Tanzania in 2015 
revealed caesarean section delivery was positively associated with postnatal care use[25]. This might be women's who gave birth by caesarean section stay at the facility for about two days for which they receive postnatal check-up.

In another way, women who gave birth at health facility were twice more likely to utilize postnatal care as compared those delivered at home. This substantiates previous findings in the study in different parts of Ethiopia like Tigray and SNNPR, [7-9, 25] and Tanzania[25]. This might be clients who visit health facility would have different health seeking behaviours with those never attended health facility.

There is significant administrative regional variation in postnatal care use in Oromia, Somali, Benishangul, SNNPR, Gambela, Afar and Dire Dawa which less likely to utilize postnatal care utilization as compared to Addis Ababa city administrative area. While there is no significant difference between residents of Harari city, Amhara regional state and Tigray regional state as compared to Addis Ababa city. Variation in postnatal care service utilization varies was also observed in different parts of Ethiopia[7, 8, 17, 18, 26] and West African countries[27]. There are several possible explanations for this finding, first the difference in geographic accessibility of the postnatal care services due to topography and unfavourable roads for the mothers in rural areas of Ethiopia; second, there were difference in local cultures and beliefs in different areas of the country; third, difference in urbanized geographical areas among different regions.

Being in the higher community level antenatal care coverage were 1.5 times more likely to utilize post-natal care services. Our findings appear to be well supported by a multilevel analysis of DHS in sub-Saharan Africa in 2014, which found significant associations between four or more antenatal care visits and ever breastfed with both outcomes[28]. The results point to the likelihood of information diffusion for postnatal care utilization in the community.

Being resident of higher community level wealth was 1.4 times more likely to utilize post-natal care services. This concurs well with the study in West Africa in 2018, which revealed 
community level poverty was a significant determinants of postnatal care use[27]. This would appear to indicate that wealthy community were more probability of getting health information and reside in urban areas.

A community who perceived the distance to health facility as a big problem were $22 \%$ less likely to utilize postnatal care as compared to their counter parts. We believe that no other authors have found that postnatal care utilization is less likely in the community who perceived the distance of health facility as a big problem. There is a good probability that community telling can influence any health care service utilization.

This study provides strong evidence in utilizing community based representative data of DHS and the use of multilevel mixed-effects analysis which bring disaggregated data on individual characteristics and community level determinants for designing contextual interventions.

We aware that our research may have two limitations. The first is findings are based on quantitative data only which cannot explore the detailed reasons in the community for low level of postnatal care use. The second is excluding men's and other community level significant others view, may not give fully address the community level determinants. These limitations are evidence of the DHS women's data not inclusive of the above problems.

\section{Conclusion}

Our work has led us to the conclusion that less than one in five women utilized postnatal care in the last two years before the survey in Ethiopia. Institutional delivery and giving birth by caesarean section were found to be individual level determinants while administrative regions, community level wealth, community level antenatal care coverage and perceived distance of the health facility as a big problem were community level determinants.

Federal ministry of health maternal health directorate should realize the equity in postnatal care services among administrative regions of the country. Public health interventions on maternal 
health should give priority for mothers never attended antenatal care visits, those who gave birth at home and who gave spontaneous vaginal delivery. Exploring community level determinants in different areas of the country using qualitative data with the inclusion male responses were recommended for scientific communities.

\section{Declarations}

336 Ethics approval and consent to participate: the EDHS program was conducted the survey

337 after getting necessary ethical reviews at country level and obtained necessary permission as an organization to conduct the survey. Privacy and confidentiality of every individuals interviewed was maintained.

340 Consent for publication and availability of data: the data was obtained after necessary agreement to publish for this research title from MEASURE DHS database at https://dhsprogram.com/data/available-datasets.cfm

Competing interests: The authors declare that they have no competing interests.

\section{Funding: N/A}

345 Authors' contributions: AT made substantial contributions to conception, design and in drafting the manuscript. MG involved acquisition of data, analysis and involved revising the manuscript critically for important intellectual content. All authors read and approved the final manuscript.

Acknowledgements: We would like to acknowledge the Demographic Health Survey program office for the realizations of this finding by authorizing the data. 
AOR: Adjusted Odds Ratio, ANC: Antenatal Care, CI: Confidence Interval, EDHS:

Class Correlation, MEASURE DHS: Monitoring and Evaluation to Assess and Use Results

Demographic and Health Surveys, PCV: Proportional Change in Variance, PNC: Postnatal

Care, SDG: Sustainable Development Goal, SNNPR: Southern Nations, Nationalities, and

Peoples' Region.

\section{References}

1. WHO: Maternal mortality: fact sheets; 2018.

2. (WHO) WHO: WHO recommendations on Postnatal care of the mother and newborn 2013. mortality. International Journal of Gynecology \& Obstetrics 1996, 54(1):1-10.

4. WHO: Technical Consultation on Postpartum and Postnatal Care. 2010.

5. Kikuchi K YJ, Nanishi K, Ahmed A, Nohara Y, Nishikitani M, et al.: Postnatal care could be the key to improving the continuum of care in maternal and child health in Ratanakiri , Cambodia. 2018:1-13.

6. CentralStatisticalAgency(CSA)[Ethiopia]andICF: Ethiopia Demographic and Health Survey 2016. In. Addis Ababa, Ethiopia, and Rockville, Maryland, USA: CSA and ICF; 2016.

7. Abraha TH, Gebrezgiabher BB, Aregawi BG, Belay DS, Tikue LT, Reda EB: Factors Associated with Compliance with the Recommended Frequency of Postnatal Care Services in Four Rural Districts of Tigray Region, North Ethiopia. Korean J Fam Med 2019.

8. Abuka Abebo T, Jember Tesfaye D: Postnatal care utilization and associated factors among women of reproductive age Group in Halaba Kulito Town, Southern Ethiopia. Arch Public Health 2018, 76:9.

9. Abota TL, Atenafu NT: Postnatal Care Utilization and Associated Factors among Married Women in Benchi-Maji Zone, Southwest Ethiopia: A Community Based Cross-Sectional Study. Ethiop J Health Sci 2018, 28(3):267-276.

10. Barry D, Frew AH, Mohammed H, Desta BF, Tadesse L, Aklilu Y, Biadgo A, Buffington ST, Sibley LM: The effect of community maternal and newborn health family meetings on type of birth attendant and completeness of maternal and newborn care received during birth and the early postnatal period in rural Ethiopia. J Midwifery Womens Health 2014, 59 Suppl 1:S44-54.

11. Chaka EE, Abdurahman AA, Nedjat S, Majdzadeh R: Utilization and Determinants of Postnatal Care Services in Ethiopia: A Systematic Review and Meta-Analysis. Ethiop J Health Sci 2019, 29(1):935-944.

12. Tesfahun F, Worku W, Mazengiya F, Kifle M: Knowledge, perception and utilization of postnatal care of mothers in Gondar Zuria District, Ethiopia: a cross-sectional study. Matern Child Health J 2014, 18(10):2341-2351.

13. Tesfaye S, Barry D, Gobezayehu AG, Frew AH, Stover KE, Tessema H, Alamineh L, Sibley LM: Improving coverage of postnatal care in rural Ethiopia using a community-based, 
collaborative quality improvement approach. J Midwifery Womens Health 2014, 59 Suppl 1:S55-64.

14. Angore BN, Tufa EG, Bisetegen FS: Determinants of postnatal care utilization in urban community among women in Debre Birhan Town, Northern Shewa, Ethiopia. J Health Popul Nutr 2018, 37(1):10.

15. Darega B, Dida N, Tafese F, Ololo S: Institutional delivery and postnatal care services utilizations in Abuna Gindeberet District, West Shewa, Oromiya Region, Central Ethiopia: A Community-based cross sectional study. BMC Pregnancy Childbirth 2016, 16:149.

16. Fekadu GA, Ambaw F, Kidanie SA: Facility delivery and postnatal care services use among mothers who attended four or more antenatal care visits in Ethiopia: further analysis of the 2016 demographic and health survey. BMC Pregnancy Childbirth 2019, 19(1):64.

17. Wudineh KG, Nigusie AA, Gesese SS, Tesu AA, Beyene FY: Postnatal care service utilization and associated factors among women who gave birth in Debretabour town, North West Ethiopia: a community- based cross-sectional study. BMC Pregnancy Childbirth 2018, 18(1):508.

18. Tesfaye G, Chojenta C, Smith R, Loxton D: Magnitude and correlates of postnatal care utilization among reproductive aged women in a rural district in eastern Ethiopia: A crosssectional study. Midwifery 2019, 70:22-30.

19. Heyi WD DM EM: Determinants of postnatal care service utilization in Diga district, East Wollega zone , Western Ethiopia : case-control study , . 2018, 10(4):52-61.

20. Iqbal S ZR, Sadaf F, Usman A. : Determinants of postnatal care services utilization in pakistan- insights from pakistan demographic and health survey (PDHS ) 2006-07. 2013 2018(January).

21. WHO: Department of Economic and Social Affairs Population Division. World Population Prospects 2019.

22. Health Mo: Health sector development program IV 2010/11-2014/15. Addis Ababa, Ethiopia: Federal Democratic Republic of Ethiopia.; 2010.

23. Barate P, Temmerman M: Why Do Mothers Die? The Silent Tragedy of Maternal Mortality. Current Women's Health Reviews 2009, 5(4):230-238.

24. Jacobs C, Moshabela M, Maswenyeho S, Lambo N, Michelo C: Predictors of Antenatal Care, Skilled Birth Attendance, and Postnatal Care Utilization among the Remote and Poorest Rural Communities of Zambia: A Multilevel Analysis. Frontiers in Public Health 2017, 5(11).

25. Mohan D, Gupta S, LeFevre A, Bazant E, Killewo J, Baqui AH: Determinants of postnatal care use at health facilities in rural Tanzania: multilevel analysis of a household survey. $B M C$ Pregnancy Childbirth 2015, 15:282.

26. Akibu M, Tsegaye W, Megersa T, Nurgi S: Prevalence and Determinants of Complete Postnatal Care Service Utilization in Northern Shoa, Ethiopia. J Pregnancy 2018, 2018:8625437.

27. Solanke BL, Amoo EO, Idowu AE: Improving postnatal checkups for mothers in West Africa: A multilevel analysis. Women \& Health 2018, 58(2):221-245.

28. Singh $\mathrm{K}$, Brodish $\mathrm{P}$, Haney $\mathrm{E}$ : Postnatal care by provider type and neonatal death in subSaharan Africa: a multilevel analysis. BMC Public Health 2014, 14:941. 